\title{
Invertible Operators on Banach Spaces
}

\author{
Kazuhisa Nakashc \\ Yamaguchi University \\ Yamaguchi, Japan
}

\begin{abstract}
Summary. In this article, using the Mizar system [2, 1, we discuss invertible operators on Banach spaces. In the first chapter, we formalized the theorem that denotes any operators that are close enough to an invertible operator are also invertible by using the property of Neumann series.

In the second chapter, we formalized the continuity of an isomorphism that maps an invertible operator on Banach spaces to its inverse. These results are used in the proof of the implicit function theorem. We referred to [3], 10, 6], 7] in this formalization.
\end{abstract}

MSC: 47A05 47J07 68T99 03B35

Keywords: Banach space; invertible operator; Neumann series; isomorphism of linear operator spaces

MML identifier: LOPBAN13, version: 8.1.09 5.57.1355

\section{Neumann Series and Invertible Operator}

From now on $X, Y, Z$ denote non trivial real Banach spaces.

Let $X, Y$ be real normed spaces and $u$ be a point of the real norm space of bounded linear operators from $X$ into $Y$. We say that $u$ is invertible if and only if

(Def. 1) $u$ is one-to-one and $\operatorname{rng} u=$ the carrier of $Y$ and $u^{-1}$ is a point of the real norm space of bounded linear operators from $Y$ into $X$.

Assume $u$ is invertible. The functor Inv $u$ yielding a point of the real norm space of bounded linear operators from $Y$ into $X$ is defined by the term (Def. 2) $u^{-1}$. 
Now we state the propositions:

(1) Let us consider a real normed space $X$, a sequence $s_{6}$ of $X$, and a natural number $k$. Then $\left\|\left(\left(\sum_{\alpha=0}^{\kappa} s_{6}(\alpha)\right)_{\kappa \in \mathbb{N}}\right)(k)\right\| \leqslant\left(\left(\sum_{\alpha=0}^{\kappa}\left\|s_{6}\right\|(\alpha)\right)_{\kappa \in \mathbb{N}}\right)(k)$.

Proof: Define $\mathcal{P}$ [natural number] $\equiv\left\|\left(\left(\sum_{\alpha=0}^{\kappa} s_{6}(\alpha)\right)_{\kappa \in \mathbb{N}}\right)\left(\$_{1}\right)\right\| \leqslant$ $\left(\left(\sum_{\alpha=0}^{\kappa}\left\|s_{6}\right\|(\alpha)\right)_{\kappa \in \mathbb{N}}\right)\left(\$_{1}\right)$. For every natural number $k, \mathcal{P}[k]$.

(2) Let us consider a real Banach space $X$, and a sequence $s$ of $X$. Suppose $s$ is norm-summable. Then $\left\|\sum s\right\| \leqslant \sum\|s\|$. The theorem is a consequence of $(1)$.

(3) Let us consider a Banach algebra, and a point $z$ of $X$. Suppose $\|z\|<1$. Then

(i) $\left(z^{\kappa}\right)_{\kappa \in \mathbb{N}}$ is norm-summable, and

(ii) $\left\|\sum\left(z^{\kappa}\right)_{\kappa \in \mathbb{N}}\right\| \leqslant \frac{1}{1-\|z\|}$.

Proof: For every natural number $n, 0 \leqslant\left\|\left(z^{\kappa}\right)_{\kappa \in \mathbb{N}}\right\|(n) \leqslant\left(\left(\|z\|^{\kappa}\right)_{\kappa \in \mathbb{N}}\right)(n)$. $\left\|\sum\left(z^{\kappa}\right)_{\kappa \in \mathbb{N}}\right\| \leqslant \sum\left\|\left(z^{\kappa}\right)_{\kappa \in \mathbb{N}}\right\|$.

(4) Let us consider a Banach algebra, and a point $w$ of $S$. Suppose $\|w\|<1$. Then

(i) $1_{S}+w$ is invertible, and

(ii) $\left((-w)^{\kappa}\right)_{\kappa \in \mathbb{N}}$ is norm-summable, and

(iii) $\left(1_{S}+w\right)^{-1}=\sum\left((-w)^{\kappa}\right)_{\kappa \in \mathbb{N}}$, and

(iv) $\left\|\left(1_{S}+w\right)^{-1}\right\| \leqslant \frac{1}{1-\|w\|}$.

The theorem is a consequence of (3).

(5) Let us consider a non trivial real Banach space $X$, Lipschitzian linear operators $v_{1}, v_{2}$ from $X$ into $X$, points $w_{1}, w_{2}$ of NormedAlgebraOfBounded$\operatorname{LinearOpers}_{\mathbb{R}}(X)$, and a real number $a$. Suppose $v_{1}=w_{1}$ and $v_{2}=w_{2}$. Then

(i) $v_{1} \cdot v_{2}=w_{1} \cdot w_{2}$, and

(ii) $v_{1}+v_{2}=w_{1}+w_{2}$, and

(iii) $a \cdot v_{1}=a \cdot w_{1}$.

PROOF: Reconsider $z_{1}=w_{1}, z_{3}=w_{2}$ as a point of the real norm space of bounded linear operators from $X$ into $X$. Reconsider $z_{2}=z_{1}+z_{3}$ as a point of the real norm space of bounded linear operators from $X$ into $X$. For every object $s$ such that $s \in \operatorname{dom}\left(v_{1}+v_{2}\right)$ holds $\left(v_{1}+v_{2}\right)(s)=z_{2}(s)$. Reconsider $z_{2}=a \cdot z_{1}$ as a point of the real norm space of bounded linear operators from $X$ into $X$. For every object $s$ such that $s \in \operatorname{dom}\left(a \cdot v_{1}\right)$ holds $\left(a \cdot v_{1}\right)(s)=z_{2}(s)$. 
(6) Let us consider a non trivial real Banach space $X$, points $v_{1}, v_{2}$ of the real norm space of bounded linear operators from $X$ into $X$, points $w_{1}, w_{2}$ of NormedAlgebraOfBoundedLinearOpers $_{\mathbb{R}}(X)$, and a real number $a$. Suppose $v_{1}=w_{1}$ and $v_{2}=w_{2}$. Then

(i) $v_{1}+v_{2}=w_{1}+w_{2}$, and

(ii) $a \cdot v_{1}=a \cdot w_{1}$.

(7) Let us consider a non trivial real Banach space $X$, points $v_{1}, v_{2}$ of the real norm space of bounded linear operators from $X$ into $X$, and points $w_{1}, w_{2}$ of NormedAlgebraOfBoundedLinearOpers $\mathbb{R}(X)$. If $v_{1}=w_{1}$ and $v_{2}=w_{2}$, then $v_{1} \cdot v_{2}=w_{1} \cdot w_{2}$.

(8) Let us consider a non trivial real Banach space $X$, a point $v$ of the real norm space of bounded linear operators from $X$ into $X$, and a point $w$ of NormedAlgebraOfBoundedLinearOpers $_{\mathbb{R}}(X)$. Suppose $v=w$. Then

(i) $v$ is invertible iff $w$ is invertible, and

(ii) if $w$ is invertible, then $v^{-1}=w^{-1}$.

Proof: If $v$ is invertible, then $w$ is invertible. If $w$ is invertible, then $v$ is invertible and $v^{-1}=w^{-1}$.

(9) Let us consider points $v, I$ of the real norm space of bounded linear operators from $X$ into $X$. Suppose $I=\operatorname{id}_{X}$ and $\|v\|<1$. Then

(i) $I+v$ is invertible, and

(ii) $\|\operatorname{Inv} I+v\| \leqslant \frac{1}{1-\|v\|}$, and

(iii) there exists a point $w$ of NormedAlgebraOfBoundedLinearOpers $\mathbb{R}_{\mathbb{R}}(X)$ such that $w=v$ and $\left((-w)^{\kappa}\right)_{\kappa \in \mathbb{N}}$ is norm-summable and Inv $I+v=$ $\sum\left((-w)^{\kappa}\right)_{\kappa \in \mathbb{N}}$.

The theorem is a consequence of (4) and (8).

(10) Let us consider real normed spaces $X, Y, Z, W$, a point $f$ of the real norm space of bounded linear operators from $X$ into $Y$, a point $g$ of the real norm space of bounded linear operators from $Y$ into $Z$, and a point $h$ of the real norm space of bounded linear operators from $Z$ into $W$. Then $h \cdot(g \cdot f)=(h \cdot g) \cdot f$.

(11) Let us consider real normed spaces $X, Y$, and a point $f$ of the real norm space of bounded linear operators from $X$ into $Y$. Suppose $f$ is one-to-one and $\operatorname{rng} f=$ the carrier of $Y$. Then

(i) $f^{-1} \cdot f=\operatorname{id}_{X}$, and

(ii) $f \cdot\left(f^{-1}\right)=\mathrm{id}_{Y}$. 
(12) Let us consider a point $u$ of the real norm space of bounded linear operators from $X$ into $Y$. Suppose $u$ is invertible. Then

(i) $0<\|u\|$, and

(ii) $0<\|\operatorname{Inv} u\|$.

(13) Let us consider points $u, v$ of the real norm space of bounded linear operators from $X$ into $Y$. Suppose $u$ is invertible and $\|v\|<\frac{1}{\|\operatorname{Inv} u\|}$. Then

(i) $u+v$ is invertible, and

(ii) $\|\operatorname{Inv} u+v\| \leqslant \frac{1}{\left\|\frac{1}{\|\operatorname{Inv} u\|}-\right\| v \|}$, and

(iii) there exists a point $w$ of NormedAlgebraOfBoundedLinearOpers $\mathbb{R}(X)$ and there exist points $s, I$ of the real norm space of bounded linear operators from $X$ into $X$ such that $w=(\operatorname{Inv} u) \cdot v$ and $s=w$ and $I=\operatorname{id}_{X}$ and $\|s\|<1$ and $\left((-w)^{\kappa}\right)_{\kappa \in \mathbb{N}}$ is norm-summable and $I+s$ is invertible and $\|\operatorname{Inv} I+s\| \leqslant \frac{1}{1-\|s\|}$ and $\operatorname{Inv} I+s=\sum\left((-w)^{\kappa}\right)_{\kappa \in \mathbb{N}}$ and $\operatorname{Inv} u+v=(\operatorname{Inv} I+s) \cdot(\operatorname{Inv} u)$.

Proof: Reconsider $I=\mathrm{id}_{X}$ as a point of the real norm space of bounded linear operators from $X$ into $X$. Reconsider $u_{1}=(\operatorname{Inv} u) \cdot v$ as a point of the real norm space of bounded linear operators from $X$ into $X$. $\|\operatorname{Inv} u\| \neq$ 0 by [9, (2)]. $I+u_{1}$ is invertible and $\left\|\operatorname{Inv} I+u_{1}\right\| \leqslant \frac{1}{1-\left\|u_{1}\right\|}$ and there exists a point $w$ of NormedAlgebraOfBoundedLinearOpers $\mathbb{R}_{(}(X)$ such that $w=u_{1}$ and $\left((-w)^{\kappa}\right)_{\kappa \in \mathbb{N}}$ is norm-summable and Inv $I+u_{1}=\sum\left((-w)^{\kappa}\right)_{\kappa \in \mathbb{N}}$. For every element $x$ of the carrier of $X,(u+v)(x)=\left(u \cdot\left(I+u_{1}\right)\right)(x)$. $\operatorname{PartFuncs}\left(\left(I+u_{1}\right)^{-1}, X, X\right)=\operatorname{PartFuncs}\left(\operatorname{Inv} I+u_{1}, X, X\right)$. Consider $w$ being a point of NormedAlgebraOfBoundedLinearOpers $\mathbb{R}_{\mathbb{R}}(X)$ such that $w=u_{1}$ and $\left((-w)^{\kappa}\right)_{\kappa \in \mathbb{N}}$ is norm-summable and Inv $I+u_{1}=\sum\left((-w)^{\kappa}\right)_{\kappa \in \mathbb{N}}$.

(14) Let us consider a subset $S$ of the real norm space of bounded linear operators from $X$ into $Y$. Suppose $S=\{v$, where $v$ is a point of the real norm space of bounded linear operators from $X$ into $Y: v$ is invertible $\}$. Then $S$ is open.

Proof: Set $P=$ the real norm space of bounded linear operators from $X$ into $Y$. For every point $u$ of $P$ such that $u \in S$ there exists a real number $r$ such that $r>0$ and $\operatorname{Ball}(u, r) \subseteq S$ by (12), [4, (17)], (13).

Let us consider $X$ and $Y$. The functor InvertOpers $(X, Y)$ yielding an open subset of the real norm space of bounded linear operators from $X$ into $Y$ is defined by the term

(Def. 3) $\{v$, where $v$ is a point of the real norm space of bounded linear operators from $X$ into $Y: v$ is invertible $\}$. 
Now we state the propositions:

(15) Let us consider a point $u$ of the real norm space of bounded linear operators from $X$ into $Y$. Suppose $u$ is invertible. Then

(i) Inv $u$ is invertible, and

(ii) $\operatorname{Inv} \operatorname{Inv} u=u$.

(16) There exists a function $I$ from $\operatorname{InvertOpers}(X, Y)$ into $\operatorname{InvertOpers}(Y, X)$ such that

(i) $I$ is one-to-one and onto, and

(ii) for every point $u$ of the real norm space of bounded linear operators from $X$ into $Y$ such that $u \in \operatorname{InvertOpers}(X, Y)$ holds $I(u)=\operatorname{Inv} u$.

Proof: Set $S=$ the real norm space of bounded linear operators from $X$ into $Y$. Define $\mathcal{Q}\left[\right.$ object, object] $\equiv$ there exists a point $u$ of $S$ such that $\$_{1}=$ $u$ and $\$_{2}=\operatorname{Inv} u$. For every object $x$ such that $x \in \operatorname{InvertOpers}(X, Y)$ there exists an object $y$ such that $y \in \operatorname{InvertOpers}(Y, X)$ and $\mathcal{Q}[x, y]$. Consider $I$ being a function from InvertOpers $(X, Y)$ into InvertOpers $(Y, X)$ such that for every object $x$ such that $x \in \operatorname{InvertOpers}(X, Y)$ holds $\mathcal{Q}[x, I(x)]$. For every point $u$ of $S$ such that $u \in \operatorname{InvertOpers}(X, Y)$ holds $I(u)=\operatorname{Inv} u$. If $\operatorname{InvertOpers}(X, Y) \neq \emptyset$, then $\operatorname{InvertOpers}(Y, X) \neq \emptyset$. For every objects $x_{1}$, $x_{2}$ such that $x_{1}, x_{2} \in \operatorname{InvertOpers}(X, Y)$ and $I\left(x_{1}\right)=I\left(x_{2}\right)$ holds $x_{1}=x_{2}$.

(17) Let us consider points $u, v$ of the real norm space of bounded linear operators from $X$ into $Y$. Suppose $u$ is invertible and $\|v-u\|<\frac{1}{\|\operatorname{Inv} u\|}$. Then

(i) $v$ is invertible, and

(ii) $\|\operatorname{Inv} v\| \leqslant \frac{1}{\|\operatorname{Inv} u\|}-\|v-u\|$, and

(iii) there exists a point $w$ of NormedAlgebraOfBoundedLinearOpers $\mathbb{R}_{\mathbb{R}}(X)$ and there exist points $s, I$ of the real norm space of bounded linear operators from $X$ into $X$ such that $w=(\operatorname{Inv} u) \cdot(v-u)$ and $s=w$ and $I=\operatorname{id}_{X}$ and $\|s\|<1$ and $\left((-w)^{\kappa}\right)_{\kappa \in \mathbb{N}}$ is norm-summable and $I+s$ is invertible and $\|\operatorname{Inv} I+s\| \leqslant \frac{1}{1-\|s\|}$ and $\operatorname{Inv} I+s=\sum\left((-w)^{\kappa}\right)_{\kappa \in \mathbb{N}}$ and $\operatorname{Inv} v=(\operatorname{Inv} I+s) \cdot(\operatorname{Inv} u)$.

The theorem is a consequence of (13). 


\section{Isomorphic Mapping to Inverse Operators}

Now we state the propositions:

(18) Let us consider real normed spaces $X, Y, Z$, a point $u$ of the real norm space of bounded linear operators from $X$ into $Y$, a point $v$ of the real norm space of bounded linear operators from $Y$ into $Z$, and a point $w$ of the real norm space of bounded linear operators from $X$ into $Z$. Suppose $w=v \cdot u$. Then $\|w\| \leqslant\|v\| \cdot\|u\|$.

(19) Let us consider real normed spaces $X, Y, Z$, points $u, v$ of the real norm space of bounded linear operators from $X$ into $Y$, and a point $w$ of the real norm space of bounded linear operators from $Y$ into $Z$. Then

(i) $w \cdot(u-v)=w \cdot u-w \cdot v$, and

(ii) $w \cdot(u+v)=w \cdot u+w \cdot v$.

Proof: For every point $x$ of $X,(w \cdot(u-v))(x)=(w \cdot u)(x)-(w \cdot v)(x)$. For every point $x$ of $X,(w \cdot(u+v))(x)=(w \cdot u)(x)+(w \cdot v)(x)$.

(20) Let us consider real normed spaces $X, Y, Z$, a point $w$ of the real norm space of bounded linear operators from $X$ into $Y$, and points $u, v$ of the real norm space of bounded linear operators from $Y$ into $Z$. Then

(i) $(u-v) \cdot w=u \cdot w-v \cdot w$, and

(ii) $(u+v) \cdot w=u \cdot w+v \cdot w$.

Proof: For every point $x$ of $X,((u-v) \cdot w)(x)=(u \cdot w)(x)-(v \cdot w)(x)$. For every point $x$ of $X,((u+v) \cdot w)(x)=(u \cdot w)(x)+(v \cdot w)(x)$.

(21) Let us consider real normed spaces $X, Y$, and points $u, v$ of the real norm space of bounded linear operators from $X$ into $Y$. Then $u-(u+v)=-v$.

(22) Let us consider real normed spaces $X, Y$, and a point $u$ of the real norm space of bounded linear operators from $X$ into $Y$. Suppose $u$ is invertible. Then

(i) $(\operatorname{Inv} u) \cdot u=\operatorname{id}_{X}$, and

(ii) $u \cdot(\operatorname{Inv} u)=\operatorname{id}_{Y}$.

(23) Let us consider a point $u$ of the real norm space of bounded linear operators from $X$ into $Y$. Suppose $u$ is invertible. Let us consider a real number $r$. Suppose $0<r$. Then there exists a real number $s$ such that

(i) $0<s$, and

(ii) for every point $v$ of the real norm space of bounded linear operators from $X$ into $Y$ such that $\|v-u\|<s$ holds $\|\operatorname{Inv} v-\operatorname{Inv} u\|<r$.

The theorem is a consequence of (12), (17), (20), (18), (22), (19), and (21). 
(24) Let us consider a partial function $I$ from the real norm space of bounded linear operators from $X$ into $Y$ to the real norm space of bounded linear operators from $Y$ into $X$.

Suppose $\operatorname{dom} I=\operatorname{InvertOpers}(X, Y)$ and for every point $u$ of the real norm space of bounded linear operators from $X$ into $Y$ such that $u \in \operatorname{InvertOpers}(X, Y)$ holds $I(u)=\operatorname{Inv} u$. Then $I$ is continuous on InvertOpers $(X, Y)$. The theorem is a consequence of $(23)$.

(25) There exists a partial function $I$ from the real norm space of bounded linear operators from $X$ into $Y$ to the real norm space of bounded linear operators from $Y$ into $X$ such that

(i) $\operatorname{dom} I=\operatorname{InvertOpers}(X, Y)$, and

(ii) $\operatorname{rng} I=\operatorname{InvertOpers}(Y, X)$, and

(iii) $I$ is one-to-one and continuous on $\operatorname{InvertOpers}(X, Y)$, and

(iv) there exists a partial function $J$ from the real norm space of bounded linear operators from $Y$ into $X$ to the real norm space of bounded linear operators from $X$ into $Y$ such that $J=I^{-1}$ and $J$ is one-to-one and $\operatorname{dom} J=\operatorname{InvertOpers}(Y, X)$ and $\operatorname{rng} J=\operatorname{InvertOpers}(X, Y)$ and $J$ is continuous on InvertOpers $(Y, X)$, and

(v) for every point $u$ of the real norm space of bounded linear operators from $X$ into $Y$ such that $u \in \operatorname{InvertOpers}(X, Y)$ holds $I(u)=\operatorname{Inv} u$.

Proof: Consider $J$ being a function from InvertOpers $(X, Y)$ into Invert$\operatorname{Opers}(Y, X)$ such that $J$ is one-to-one and onto and for every point $u$ of the real norm space of bounded linear operators from $X$ into $Y$ such that $u \in \operatorname{InvertOpers}(X, Y)$ holds $J(u)=\operatorname{Inv} u$. If $\operatorname{InvertOpers}(X, Y) \neq \emptyset$, then InvertOpers $(Y, X) \neq \emptyset$. Reconsider $L=J^{-1}$ as a function from $\operatorname{InvertOpers}(Y, X)$ into InvertOpers $(X, Y)$. For every point $v$ of the real norm space of bounded linear operators from $Y$ into $X$ such that $v \in$ $\operatorname{InvertOpers}(Y, X)$ holds $L(v)=\operatorname{Inv} v$.

Let us consider real normed spaces $X, Y, Z$, a point $u$ of the real norm space of bounded linear operators from $X$ into $Y$, and a point $w$ of the real norm space of bounded linear operators from $Y$ into $Z$. Now we state the propositions:

(i) $w \cdot(-u)=-w \cdot u$, and

(ii) $(-w) \cdot u=-w \cdot u$.

Proof: For every point $x$ of $X,(w \cdot(-u))(x)=(-1) \cdot(w \cdot u)(x)$. For every point $x$ of $X,((-w) \cdot u)(x)=(-1) \cdot(w \cdot u)(x)$.

(27) $(-w) \cdot(-u)=w \cdot u$. The theorem is a consequence of $(26)$.

(28) Let us consider real normed spaces $X, Y, Z$, a point $u$ of the real norm space of bounded linear operators from $X$ into $Y$, a point $w$ of the real 
norm space of bounded linear operators from $Y$ into $Z$, and a real number $r$. Then

(i) $w \cdot(r \cdot u)=(r \cdot w) \cdot u$, and

(ii) $r \cdot w \cdot u=r \cdot w \cdot u$, and

(iii) $(r \cdot w) \cdot u=r \cdot(w \cdot u)$.

Proof: For every point $x$ of $X,(w \cdot(r \cdot u))(x)=r \cdot(w \cdot u)(x)$. For every point $x$ of $X,(r \cdot w \cdot u)(x)=r \cdot(w \cdot u)(x)$.

(29) Let us consider real normed spaces $X, Y, Z$. Then there exists a bilinear operator $I$ from the real norm space of bounded linear operators from $X$ into $Y \times$ the real norm space of bounded linear operators from $Y$ into $Z$ into the real norm space of bounded linear operators from $X$ into $Z$ such that

(i) $I$ is continuous on the carrier of (the real norm space of bounded linear operators from $X$ into $Y) \times($ the real norm space of bounded linear operators from $Y$ into $Z$ ), and

(ii) for every point $u$ of the real norm space of bounded linear operators from $X$ into $Y$ and for every point $v$ of the real norm space of bounded linear operators from $Y$ into $Z, I(u, v)=v \cdot u$.

Proof: Set $E=$ the real norm space of bounded linear operators from $X$ into $Y$. Set $F=$ the real norm space of bounded linear operators from $Y$ into $Z$. Set $G=$ the real norm space of bounded linear operators from $X$ into $Z$. Define $\mathcal{Q}[$ object, object] $\equiv$ there exists a point $u$ of $E$ and there exists a point $v$ of $F$ such that $\$_{1}=\langle u, v\rangle$ and $\$_{2}=v \cdot u$. For every object $x$ such that $x \in$ the carrier of $E \times F$ there exists an object $y$ such that $y \in$ the carrier of $G$ and $\mathcal{Q}[x, y]$ by [5, (18)]. Consider $L$ being a function from the carrier of $E \times F$ into the carrier of $G$ such that for every object $x$ such that $x \in$ the carrier of $E \times F$ holds $\mathcal{Q}[x, L(x)]$.

For every point $u$ of the real norm space of bounded linear operators from $X$ into $Y$ and for every point $v$ of the real norm space of bounded linear operators from $Y$ into $Z, L(u, v)=v \cdot u$. For every points $x_{1}, x_{2}$ of $E$ and for every point $y$ of $F, L\left(x_{1}+x_{2}, y\right)=L\left(x_{1}, y\right)+L\left(x_{2}, y\right)$. For every point $x$ of $E$ and for every point $y$ of $F$ and for every real number $a$, $L(a \cdot x, y)=a \cdot L(x, y)$. For every point $x$ of $E$ and for every points $y_{1}, y_{2}$ of $F, L\left(x, y_{1}+y_{2}\right)=L\left(x, y_{1}\right)+L\left(x, y_{2}\right)$. For every point $x$ of $E$ and for every point $y$ of $F$ and for every real number $a, L(x, a \cdot y)=a \cdot L(x, y)$. Set $K=1$. For every point $x$ of $E$ and for every point $y$ of $F,\|L(x, y)\| \leqslant K \cdot\|x\| \cdot\|y\|$. 
Let us consider real normed spaces $X, Y$, a Lipschitzian linear operator $v$ from $X$ into $Y$, a point $w$ of the real norm space of bounded linear operators from $X$ into $Y$, and a real number $a$. Now we state the propositions:

(30) If $v=w$, then $a \cdot w=a \cdot v$.

ProOF: For every object $s$ such that $s \in \operatorname{dom}(a \cdot v)$ holds $(a \cdot v)(s)=$ $(a \cdot w)(s)$ by $[8,(36)]$.

(31) If $v=w$, then $-w=-v$. The theorem is a consequence of (30).

ACKNowledgement: I would like to express my gratitude to Professor Yasunari Shidama for his helpful advice.

\section{REFERENCES}

[1] Grzegorz Bancerek, Czesław Byliński, Adam Grabowski, Artur Korniłowicz, Roman Matuszewski, Adam Naumowicz, and Karol Pąk. The role of the Mizar Mathematical Library for interactive proof development in Mizar Journal of Automated Reasoning, 61(1):9-32, 2018. do1:10.1007/s10817-017-9440-6

[2] Adam Grabowski, Artur Korniłowicz, and Adam Naumowicz. Four decades of Mizar. Journal of Automated Reasoning, 55(3):191-198, 2015. doi $10.1007 / \mathrm{s} 10817-015-9345-1$

[3] Miyadera Isao. Functional Analysis. Riko-Gaku-Sya, 1972.

[4] Kazuhisa Nakasho, Yuichi Futa, and Yasunari Shidama. Implicit function theorem. Part I. Formalized Mathematics, 25(4):269-281, 2017. doi:10.1515/forma-2017-0026.

[5] Hiroyuki Okazaki, Noboru Endou, and Yasunari Shidama. Cartesian products of family of real linear spaces. Formalized Mathematics, 19(1):51-59, 2011. doi:10.2478/v10037011-0009-2.

[6] Laurent Schwartz. Théorie des ensembles et topologie, tome 1. Analyse. Hermann, 1997.

[7] Laurent Schwartz. Calcul différentiel, tome 2. Analyse. Hermann, 1997.

[8] Yasunari Shidama. Banach space of bounded linear operators Formalized Mathematics, 12(1):39-48, 2004.

[9] Yasunari Shidama. The Banach algebra of bounded linear operators Formalized Mathematics, 12(2):103-108, 2004.

[10] Kosaku Yoshida. Functional Analysis. Springer, 1980.

Accepted May 27, 2019 